Revue d'histoire de l'Amérique française

REVUE D.HISTOIRE DE L'AMÉRIQUE FRANÇAISE

\title{
N’être plus la déléguée de personne : une réévaluation du rôle des femmes dans le commerce en Nouvelle-France
}

\section{Jan Noel}

Volume 63, numéro 2-3, automne-hiver 2009-2010

Femmes, culture et pouvoir

URI : https://id.erudit.org/iderudit/044453ar

DOI : https://doi.org/10.7202/044453ar

Aller au sommaire du numéro

\section{Éditeur(s)}

Institut d'histoire de l'Amérique française

ISSN

0035-2357 (imprimé)

1492-1383 (numérique)

Découvrir la revue

Citer cet article

Noel, J. (2009). N'être plus la déléguée de personne : une réévaluation du rôle des femmes dans le commerce en Nouvelle-France. Revue d'histoire de l'Amérique française, 63(2-3), 209-241. https://doi.org/10.7202/044453ar
Résumé de l'article

Les historiens ont eu tendance à réduire les femmes engagées dans le commerce en Nouvelle-France à une poignée de chefs d'entreprise autonomes opérant à grande échelle. La recherche a révélé que ces individus exceptionnels étaient flanqués d'un groupe un peu plus nombreux qui ne jouissait pas de la même indépendance, comptant des femmes déléguées par leurs maris ou des veuves de marchands reprenant l'entreprise du défunt. Cet article offre un survol des livres de compte de la traite des fourrures et de l'économie de guerre, identifiant de nombreuses fournisseuses. Il affirme donc que la mise en marché de biens et de services était une activité quotidienne de toutes sortes de femmes - jeunes filles, épouses, veuves, nonnes - qui en avaient, pour l'essentiel, la direction et qui les combinaient régulièrement à leurs autres tâches.
Tous droits réservés @ Institut d'histoire de l'Amérique française, 2010
Ce document est protégé par la loi sur le droit d'auteur. L'utilisation des services d'Érudit (y compris la reproduction) est assujettie à sa politique d'utilisation que vous pouvez consulter en ligne.

https://apropos.erudit.org/fr/usagers/politique-dutilisation/ 


\title{
N'être plus la déléguée de personne: une réévaluation du rôle des femmes dans le commerce en Nouvelle-France
}

\author{
JAN NOEL \\ Département d'histoire \\ Université de Toronto
}

\begin{abstract}
RÉSUMÉ • Les historiens ont eu tendance à réduire les femmes engagées dans le commerce en Nouvelle-France à une poignée de chefs d'entreprise autonomes opérant à grande échelle. La recherche a révélé que ces individus exceptionnels étaient flanqués d'un groupe un peu plus nombreux qui ne jouissait pas de la même indépendance, comptant des femmes déléguées par leurs maris ou des veuves de marchands reprenant l'entreprise du défunt. Cet article offre un survol des livres de compte de la traite des fourrures et de l'économie de guerre, identifiant de nombreuses fournisseuses. II affirme donc que la mise en marché de biens et de services était une activité quotidienne de toutes sortes de femmes - jeunes filles, épouses, veuves, nonnes - qui en avaient, pour l'essentiel, la direction et qui les combinaient régulièrement à leurs autres tâches.
\end{abstract}

ABSTRACT • Historians have tended to portray women who were involved in commerce in New France as a handful of large-scale, self-motivated entrepreneurs. The literature presents these exceptional individuals as flanked by a somewhat larger group that was less independent, consisting of wives deputized by their husbands or merchants' widows stepping into the dead man's shoes. This article reviews fur trade and wartime accounts and finds a host of female provisioners. It makes the case that presenting services and commodities in the marketplace was an everyday, largely self-directed activity which all sorts of women girls, wives, widows and even nuns - conducted on a regular basis alongside their other tasks. 
... a wife was a dependent ${ }^{1}$... ... le rôle de représentation des femmes est très limité, peu importe le domaine d'activité. Ce rôle est surtout le fait de quelques femmes de familles marchandes particulièrement actives ${ }^{2}$... ... correspondence offers us an insight into the business and personal relationship of ... female associates (and) [...] connections between women in

Quebec and Montreal ${ }^{3}$. "deputy husband"... describe(s) an aspect of the married woman's role in colonial New England. It was considered normal [...] for a wife to assume the position of head of household in an emergency or when the husband was absent or dead. [...] day-to-day business affairs of the family were probably run in New France much as they were in [... New England ${ }^{4}$. ...they distinguish themselves as femmes favorisées ${ }^{5}$.

Comme le suggèrent les citations ci-dessus, les historiens qui ont traité des femmes en Nouvelle-France les représentent comme dépendantes des hommes, à l'exception des très rares femmes s'adonnant au commerce. Ces dernières étaient perçues comme des anomalies. Chacune devait pouvoir compter sur un réseau de femmes ayant la même tournure d'esprit pour la soutenir. Elle ne sortait de son rôle habituel que lorsque son mari lui déléguait temporairement des fonctions qui lui revenaient d'ordinaire. De telles personnes, selon une autre école, étaient "favorisées» par rapport aux femmes d'autres cultures.

Bien que toutes ces affirmations contiennent une part de vérité, elles tendent à détourner l'attention de l'expérience quotidienne et ordinaire des femmes engagées dans le «commerce». Celle-ci était tout à la fois plus réduite et plus étendue que ne le suggèrent les commentaires ci-dessus. Elle était plus réduite en ce sens que les personnes concernées étaient le plus souvent d'humble condition et tendaient à s'y livrer d'une matière

1. Peter Moogk, La Nouvelle-France: The Making of French Canada-A Cultural History (East Lansing, Michigan State University Press, 2000), 161, affirmation répétée p. 207.

2. Josette Brun, Vie et mort du couple en Nouvelle-France: Québec et Louisbourg au XvIII siècle (Montréal, McGill-Queen's, 2006), 33. L'auteure reconnaît qu’en dépit du manque de signatures féminines dans les actes notariés, «la collaboration des femmes au quotidien [...] on [la] devine plus importante que sur la scène publique.»

3. Kathryn Young, “ “... sauf les perils et fortunes de la mer”: Merchant Women in New France and the French Transatlantic Trade, 1713-1746», Canadian Historical Review, 77, 3 (1996): 405.

4. Allan Greer, The People of New France (Toronto, University of Toronto Press, 1997), 68, 70.

5. Jan Noel, «New France: Les Femmes Favorisées», dans V. Strong-Boag \& Anita Clair Fellman, dir., Rethinking Canada: The Promise of Women's History (Toronto/New York, Oxford University Press, 1997), 51. Première publication dans Atlantis, 6, 2 (1981). 
intermittente au lieu d'en faire une sorte de choix de carrière. Par contre, elle était plus étendue que ne l'indiquent les études car, loin d'être exceptionnelles ou anormales, les négociantes étaient nombreuses; vraisemblablement, c'était même la majorité des femmes qui prenait part aux échanges commerciaux. Toutefois, si les textes traitant de la NouvelleFrance se sont intéressés à plusieurs douzaines de négociantes de premier plan, ils n'offrent que de brefs aperçus des innombrables négociantes occasionnelles ou de moindre envergure ${ }^{6}$.

Il existe des moyens de révéler ce groupe occulté. Deux secteurs de l'économie fournissent des indices sur la fréquence de la commercialisation de biens et de services par des femmes. Tout d'abord, d'innombrables femmes s'investirent dans la traite des fourrures, principale source d'exportations de la Nouvelle-France. Beaucoup d'autres se mobilisèrent pour fournir des produits et services au gouvernement en temps de guerre, circonstance qui n'était que trop fréquente dans la colonie. Après une brève revue des thèmes historiographiques concernant les femmes et le commerce aux débuts du Canada et dans les colonies voisines, nous explorerons les preuves empiriques.

\section{DES FEMMES D'AFFAIRES AUX COLONIES : LES COURANTS HISTORIOGRAPHIQUES}

Le livre d'Alice Clark, Working Life of Women in the Seventeenth Century ${ }^{7}$, publié pour la première fois à Londres en 1919, posa un jalon dans l'histoire de la femme moderne. Présentant une histoire économique diversifiée qui transcendait les sempiternelles tâches domestiques, l'ouvrage

6. Par exemple, quelque trente-six commerçantes apparaissent dans «... Sauf les perils...» de Young, cependant qu'il existe des travaux valables sur des individus, tels que: Lillianne Plamondon, "Une femme d'affaires en Nouvelle-France: Marie-Anne Barbel, veuve Fornel», Revue d'histoire de l'Amérique française, 31,2 (septembre 1977): 165-185, et Lorraine Gadoury, «Une famille noble en Nouvelle-France: les D’Ailleboust ", thèse de maîtrise, Université de Montréal, 1982. Dans un texte qui a remarquablement résisté à l'usure du temps, Micheline Dumont et al., L'Histoire des femmes au Québec depuis quatre siècles (Montréal, Les Quinze, 1982), les auteures notèrent que «comme la distinction entre les sphères privée et publique n'est pas rigide, les affaires familiales, économiques et politiques ont souvent tendance à s'entremêler. Femmes et hommes s'en mêlent. » (129) C'est probablement l'absence d'études sur le petit commerce féminin qui a fait que l'ouvrage insiste sur les longues et pénibles corvées domestiques comme la cuisine et le ménage, conjuguées avec les travaux de la ferme. Dans cet article, nous tentons d'apporter un fondement empirique à ce commentaire éclairé du livre: «La fortune familiale détermina si elle doit participer aux activités de son époux ou faire des travaux d'appoint, couture ou lavage, pour boucler le budget.» (111) Cependant, nous pensons pouvoir élargir le débat un peu au-delà de l'affirmation qui l'accompagne : «Le travail d'une femme, qu'elle soit noble ou paysanne, se fait dans le cadre des activités définies par son époux.»

7. Alice Clark, Working Life of Women in the Seventeenth Century (Londres, Routledge, 1919), $335 \mathrm{p}$. 
démontrait le rôle productif essentiel des femmes anglaises au début de l'époque moderne. Les archives des guildes, du XIv ${ }^{e}$ au XvII siècle, pour les charpentiers, les armuriers, les tapissiers, les apothicaires, les orfèvres et les marchands, mentionnaient les «frères et sœurs " à l'œuvre dans ces négoces et exhortaient les apprentis à obéir à leurs "maîtres et maîtresses". D’autres documents identifiaient des femmes qui géraient des biens immobiliers, dirigeaient des laiteries, organisaient des vergers et des jardins, travaillaient comme soignantes et sages-femmes, possédaient des patentes et pratiquaient toutes sortes de métiers, de l'affréteuse prospère à l'humble colporteuse transportant sur son dos des ballots ou paniers sur de grandes distances. Clark faisait remarquer que, partout en Angleterre, les femmes non seulement cuisinaient mais produisaient une grande partie de la nourriture familiale. Elles fabriquaient également les vêtements. Les épouses, concluait-elle, "can hardly have been regarded as mere dependants on their husbands when the clothing for the whole family was spun by their hands ${ }^{8} »$.

La situation s'est détériorée, selon Clark, quand l'organisation capitaliste a commencé à transformer la production textile, dans la seconde moitié du XviI ${ }^{\mathrm{e}}$ siècle. La pauvreté a forcé certaines femmes à accepter les bas salaires offerts par les employeurs. La perte d'un rôle direct dans la production domestique les a marginalisées. Vers la même époque, les Anglaises en général ont fait face aux changements idéologiques qui ont réduit leurs possibilités de s'éduquer et de recevoir une formation scientifique ou professionnelle.

Working Life of Women in the Seventeenth Century a été réimprimé en 1968, 1982 et encore en 1993 pour un public avide de jeunes universitaires féministes de part et d'autre de l'Atlantique, et il a grandement influencé le domaine naissant de l'histoire des femmes. Il a contribué à accréditer l'idée que la marginalisation économique des femmes remontait au début de la période moderne, quelque temps après 1600 . Les critiques appellent cela la théorie de «l'âge d'or», mais comme ce choix de mots en fait d'emblée un mythe, on peut y référer de manière plus neutre comme la théorie de la "marginalisation». Le concept a débordé sur d'autres périodes, créant une tendance générale dans les études féministes à opposer des histoires "régressives» aux histoires "progressistes ${ }^{9}$ ".

8. A. Clark, Working Life of Women in the Seventeenth Century (Londres, Cass, 1968 [1919], d'où sont tirées les citations de Clark utilisées ici), 145.

9. La diversité des occupations suggérée par les travaux de Clark fut ensuite confirmée par les études de: Bridget Hill, Women, Work, and Sexual Politics in Eighteenth Century England (Oxford, 
Les Canadiens ont été sensibles à ces courants. L'idée selon laquelle les femmes disposaient d'une plus grande autonomie dans le passé a certainement influencé ma réflexion lorsque, étudiante diplômée dans les années 1980, j'ai lu la documentation remarquable au sujet des femmes en Nouvelle-France: Marie de l'Incarnation, Jeanne Mance et les autres dévotes qui firent tant pour la fondation de la colonie; les guerrières fougueuses comme Madame de La Tour et Madeleine de Verchères, qui défendirent des forts; et les énergiques seigneuresses comme Louise de Ramezay et Agathe de Saint-Père, qui établirent des moulins et des fabriques. Leurs activités contrastaient avec l'existence domestique plus circonscrite des Canadiennes françaises victoriennes dont les initiatives publiques se cantonnaient le plus souvent aux œuvres éducatives et de charité, qui ne fondèrent certes pas de colonies, ne gérèrent pas de grandes entreprises, ni ne prirent les armes. Sur les traces des femmes d'Alice Clark, ces femmes du Régime français, moins inhibées, illustrent fièrement mon article "New France: les femmes favorisées", paru à l'origine dans la revue Atlantis en 1981.

Le travail s'est poursuivi alors, et il se poursuit encore. Lillianne Plamondon et Micheline D’Allaire ont rédigé des études novatrices sur les entreprises au Labrador de la veuve Fornel et les pratiques commerciales avisées des ordres religieux féminins, qui étaient de très grands propriétaires fonciers. Micheline Dumont, l'une des pionnières de l'histoire des femmes au Québec, a mis en garde contre un optimisme excessif, mais a conclu, dans un texte marquant, publié en 1982 avec ses collègues, L'histoire des femmes au Québec depuis quatre siècles, que "Tout compte fait, la vie des femmes, même accaparée par de nombreuses maternités, déborde largement l'univers des langes et des enfants, car dans l'Ancien Régime, les femmes occupent une place qui nous semble aujourd'hui considérable ${ }^{10}$.» Cela dit, les auteures de ce manuel essentiel fournissaient peu de détails sur les activités économiques des citadines qui ne faisaient pas partie de l'élite, car les recherches faisaient défaut. Même les investigations méritoires qui se sont intéressées plus récemment aux femmes dans les actes légaux écartent trop rapidement les affirmations des officiels selon qui les femmes

Basil Blackwell, 1989), et de Peter Earle, "The female labour market in London in the seventeenth and early eighteenth centuries ", dans Pamela Sharp, dir., Women's Work: The English Experience, 1650-1914 (Londres, Arnold, 1998), 121-147. Selon l'étude d'Earle, qui porte sur un échantillon londonien de 1695 à 1725 , tandis que $28 \%$ des femmes déclaraient avoir été payées un salaire à Londres en 1851 , ce sont $57 \%$ des femmes de 1695-1725 qui rapportaient la même chose.

10. M. Dumont et al., op. cit., 98. 
étaient actives dans le commerce, et laissent de côté les nombreuses négociantes modestes, occasionnelles ou clandestines, qui n'auraient jamais fréquenté l'étude d'un notaire. Cela tend à créer l'impression trompeuse que les femmes étaient des participantes négligeables à l'économie de marché et que "selon toute vraisemblance, la plupart des femmes sont tenues, à divers degrés, à l'écart des affaires de leur société conjugale ${ }^{11}$ ».

Heureusement, la recherche sur les entreprises des Canadiennes d'autrefois bénéficie d'un regain de vie, comme en témoigne ce numéro de la Revue d'histoire de l'Amérique française, et nous procure une documentation plus fournie sur leur travail dans les villes. Il nous reste beaucoup à apprendre sur les raisons de son dépérissement sous le Régime britannique. Peut-être viendrons-nous même à bout de l'ultime bastion: l'hypothèse voulant que tandis que les femmes accomplissaient des tâches variées, les hommes dédaignaient les corvées féminines. Si Jacques apprit en voyageant à faire la cuisine au gré des haltes, s'empêchait-il réellement de chercher de l'eau, de s'occuper du bébé ou de touiller le pot-au-feu quand sa Jeanne était malade ou prise ailleurs?

Tandis que les années 1970 et les suivantes ont vu un intérêt renouvelé pour les femmes en Nouvelle-France, les universitaires américaines ont concentré leur attention sur les femmes des colonies voisines de la Nouvelle-Angleterre et de l'État de New York. Impressionnés par les épouses qui faisaient du commerce transatlantique depuis l'ancien Manhattan et par les patronnes de vastes domaines coloniaux, ils ont aussi été influencés par la question de la marginalisation soulevée par Alice Clark. Ils ont exploré comment certaines femmes de l'ère coloniale occupaient une plus grande variété d'emplois, exerçaient une plus grande influence économique et jouissaient d'une plus grande présence civique que leurs propres petites-filles.

Les historiennes américaines des colonies du nord, qui jouxtaient le Canada, ont envisagé d'autres aspects de la question. Gloria Main croyait qu'en fait les choses s'étaient améliorées pour les femmes de la NouvelleAngleterre au cours de la période coloniale. Elle a identifié quatre périodes économiques dans leur histoire. Il y a d'abord eu la période des pionniers, durant laquelle les deux sexes travaillaient dur à l'extérieur, côte à côte, suivie d'une époque marquée par un accroissement de la production domestique féminine et des échanges, à la fin du XvII siècle. Après 1715 ,

11. J. Brun, op. cit., 34. Cette étude soutient toutefois que les veuves étaient nettement plus actives dans le commerce que les épouses. 
on note une demande croissante pour le travail qualifié non agricole des deux sexes. La quatrième période (1739-1760), marquée par l'émigration, la croissance économique et la guerre contre les Français, amène encore plus de paysannes au travail salarié. Le statut des femmes et le cycle économique régional s'en trouvent améliorés, associés à une croissance de la proportion de jeunes femmes travaillant hors de chez elles durant les dernières décennies de la période coloniale ${ }^{12}$.

D’autres ont surtout perçu la réduction des possibilités indiquée par Clark. Elaine Foreman Crane soutient que, bien que les gages des femmes de Nouvelle-Angleterre aient été constamment inférieurs à ceux des hommes, cela comptait moins dans l'économie de troc des débuts de la période coloniale, quand les femmes échangeaient leurs tissus et autres produits contre d'autres biens. Elle trouve des veuves et des épouses jouant un rôle important dans les livres de comptes du XviI ${ }^{\mathrm{e}}$ siècle, mais moins dans ceux du xvirI ${ }^{\mathrm{e}}$ siècle. Quant au futur État de New York, Carol Berkin recense quarante-six négociantes à Albany, peu après 1650, mais aucune en 1700, cependant que la ville de New York les voit aussi diminuer dans les rangs des marchands et pratiquement disparaittre en tant qu'aubergistes, brasseuses, blanchisseuses et boulangères ${ }^{13}$.

Deborah Rosen relève elle aussi que le nombre de femmes parmi les marchands, boutiquiers et artisans chute durant la période coloniale anglaise, l'attribuant au remplacement graduel des lois et coutumes hollandaises en usage à New York par les pratiques plus restrictives des Anglais. L’usage croissant du crédit durant la révolution consumériste du $\mathrm{XVIII}^{\mathrm{e}}$ siècle gêne davantage les femmes, qui ne pouvaient signer de contrats exécutoires ${ }^{14}$. Gloria Main elle-même concède qu'il y avait davantage d'égalité entre les sexes aux premiers temps de la colonisation quand les tâches des hommes et des femmes tendaient à se confondre ${ }^{15}$. Il se peut donc qu'il y ait eu plus d'égalité entre les sexes dans le contexte plus rude du $\mathrm{XvIII}^{\mathrm{e}}$ siècle même si la prospérité générale aurait été plus grande au $\mathrm{XVII}^{\mathrm{e}}$ siècle et aurait profité à tous.

12. Gloria Main, "Gender, Work and Wages in Colonial New England», William and Mary Quarterly, 3e série, LI,1 (1994): 65.

13. Carol Berkin, First Generations: Women in Colonial America (New York, Hill and Wang, 1996), 87.

14. Deborah Rosen, «Women and Property accross Colonial America: A Comparison of Legal Systems in New Mexico and New York", William and Mary Quarterly, $3^{\text {e }}$ série, LX,2 (2003): 377 ; Deborah Rosen, Courts and Commerce: Gender, Law and the Market Economy in Colonial New York (Columbus, Ohio State University Press, 1997), 232 p.

15. G. Main, loc. cit., 54. 
Une voix plutôt conservatrice a voulu laisser sur la touche le débat sur l'amélioration ou la dégradation des choses. Laurel Ulrich, dans un ouvrage récompensé par le prix Pulitzer, Good Wives: Image and Reality in the Lives of Women in Northern New England 1650-1750, écrit:

The story of female experience in America is not to be found in a linear progression from darkness into light, from constricted to expanding opportunities....(or vice versa), but in a convoluted and sometimes tangled embroidery of loss and gain, accommodation and resistance....[S]tatus is... so complex. To enlarge the role of deputy husband might mean to contract the often highly cherished roles of housekeeper and mother ${ }^{16}$.

Ulrich esquive l'affrontement entre un camp d'historiens qui conçoit l'ère coloniale comme une ère de liberté pour les femmes qui travaillaient comme forgeronnes, ferblantières, armurières, imprimeuses ou charpentières de marine, et un autre camp qui voit des femmes strictement confinées aux soucis domestiques (telles les femmes loyalistes étudiées par Mary Beth Norton, incapables de décrire les possessions de leur famille). Ulrich le réduit à rien de plus que la différence entre ce qui était permis et ce qui était probable. La majorité des épouses en NouvelleAngleterre était, déclare-t-elle, par définition des ménagères avant tout ${ }^{17}$. Ce rôle mériterait plus de respect de la part des historiens: rester à la maison en compagnie d'un mari dont les décisions étaient censées tenir compte des opinions et intérêts de son épouse n'était pas restrictif mais noble. Les compétences de l'épouse comprenaient l'abattage des cochons, la fabrication de chandelles et, bien sûr, l'éternel filage au rouet. Le chefd'œuvre de la femme était sa descendance et son maternage s'étendait à tout le voisinage sur plusieurs générations.

L’aspect le plus controversé de Good Wives, c’est le concept de «déléguée du mari». Les femmes qui jouaient des rôles que le $\mathrm{xIx}^{\mathrm{e}}$ siècle devait qualifier de "masculins", comme de gérer des fermes ou des entreprises, ou qui exerçaient un métier ou un artisanat plus communément fait par les hommes, ne sont jamais perçues par Ulrich comme le faisant sur un pied d'égalité ou de manière indépendante. Elle affirme que la notion d'indépendance féminine était étrangère à cette culture centrée sur la famille: "Almost any task was suitable for a woman as long as it furthered the good of her family and was acceptable to her husband.» Il est vrai qu'une femme

16. Voir Laurel Thatcher Ulrich, Good Wives: Image and Reality in the Lives of Women in Northern New England, 1650-1750 (New York, Vintage, 1991), 240-241.

17. Ibid., 36. 
douée pour les affaires pouvait devenir une sorte de double de son mari, mais la plupart des épouses, rappelle Ulrich au lecteur, avaient bien autre chose à faire ${ }^{18}$. Si nous nous attardons aux idées d'Ulrich, c'est parce qu'elles ont eu beaucoup d'influence en Amérique et aussi parce qu'on a suggéré qu'elles pouvaient aider à comprendre les Canadiennes françaises d'autrefois ${ }^{19}$. Pourtant il semble tautologique d'assigner par définition les femmes à un rôle de "déléguées» sans référence aux chiffres réels ou aux circonstances qui les sous-tendent. Cet édifice conceptuel pose également la question des nombreuses non-épouses - célibataires à vie ou veuves pendant des décennies - qui travaillaient dans le négoce et le commerce.

En tout cas, les femmes au Canada, comme leurs voisines du New York hollandais, étaient moins tributaires de leur mari que ne l'étaient leurs homologues anglo-américaines soumises à la Common Law. Les femmes issues des cultures de l'Europe du Nord étaient mieux équipées pour devenir négociantes. Quand ils arrivèrent dans la première moitié du XVII ${ }^{\mathrm{e}}$ siècle, les colons français et hollandais apportèrent des codes de lois qui étaient relativement respectueux des droits de propriété des femmes. Dans les deux cultures, on s'attendait à ce que les filles comme les fils héritent de parts égales ou suffisantes de la propriété familiale. Au sein du mariage, bien que le mari ait un pouvoir de gestion, il existait une "communauté de biens » considérée comme la propriété conjointe des époux plutôt que celle du seul mari; et la veuve héritait de la moitié du tout en usufruit viager. Les épouses françaises pouvaient faire légalement des affaires en autant qu'elles eussent la permission écrite de leur mari; dans le système hollandais, une simple permission verbale suffisait. Tandis que les spécialistes du New York hollandais y voient des traditions agréablement égalitaires, les spécialistes de la Nouvelle-France se demandent toujours dans quelle mesure les protections formelles créaient des conditions favorables en pratique ${ }^{20}$. Pourtant, personne ne remet en question que ces deux

18. Ibid., 37-43. L'expression «deputy husband» (déléguée du mari) n’apparait dans Good Wives qu'une seule fois dans les citations abondantes de sources de première main, sous la forme d'une référence unique dans Ornaments for the Daughters of Zion, de Coton Mather en 1692 (249n). La "déléguée du mari» est donc une abstraction et rien ne prouve que cette expression ait fait partie de la langue courante.

19. A. Greer, op. cit., 68-70.

20. Sur la loi hollandaise dans les Nouveaux Pays-Bas et New York, voir: David Narrett, Inheritance and Family Life in Colonial New York (Ithaca, Cornell University Press, 1992), spécialement 48, 103, 207; voir aussi Linda Biemer, "Criminal Law and Women in New Amsterdam and Early New York", dans N. Zeller et C. Gehring, dir., A Beautiful and fruitful place: Selected Rensselaerswijck Seminar Papers (Albany, New Netherlands Publishing, 1991), 73-76. Sur les pratiques hollandaises du continent, voir: Danielle van den Heuvel, Women and Entrepreneurship: Female Traders in the 
systèmes continentaux gagnaient à être comparés à la Common Law, sous laquelle les épouses perdaient leur personnalité juridique et les veuves étaient parmi les moins protégées du monde ${ }^{21}$. Ces codes plus équitables survécurent aux changements de régimes dans les deux régions, subsistant longtemps après la conquête par les Anglais de la Nouvelle-Hollande en 1664 et de la Nouvelle-France en 1760.

\section{LA TRAITE DES FOURRURES}

En nous intéressant maintenant à la traite des fourrures, nous aborderons non seulement le cas canadien, mais aussi celui des Hollandais et des Iroquois (ou, pour utiliser le nom qu'ils se donnent, les Haudénosaunis) qui pratiquèrent le même commerce. Les spécialistes de la colonie hollandaise nous informent qu'en de nombreuses occasions, il y a eu une participation féminine durant le dernier quart du $\mathrm{xvII}^{\mathrm{e}}$ siècle dans la région d'Albany et de Schenectady. Beaucoup travaillaient en marge de la loi, cherchant à éluder les restrictions sur l'alcool et les taxes qui finançaient le logement des Autochtones durant les séjours de traite l'été. Il n'était pas rare pour une telle négociante de plaider l'indigence, échangeant de la boisson contre des castors parce qu'elle avait besoin de leur chair pour

Northern Netherlands c. 1580-1815 (Amsterdam, Askant, 2007), 334 p., et pour le contexte légal voir Jan Bosch, «Le statut de la Femme dans les anciens Pays-Bas septentrionaux», Recueils de la Société Jean Bodin: La Femme, $2^{\mathrm{e}}$ partie (Bruxelles, Librairie encyclopédique, 1962), 323-350. Je dois l’information sur les permissions verbales autorisant les Hollandaises à négocier à la présentation de Kim Todt: «Maria van Rensselaer and the Trading Women of New Netherlands» (Université de Sherbrooke, Colloque Femmes, Culture et Pouvoir, Sherbrooke, Québec, mai 2009). Biemer note que les quarante-six négociantes d'Albany durant la dernière décennie de la loi hollandaise déclinèrent en nombre sous la loi anglaise, n'étant plus que six en 1685-1694. Voir aussi Carole Shammas, "Anglo-American Household Government in Comparative Perspective», William and Mary Quarterly, $3^{\text {e }}$ série, LII,1 (1995): 104-144. Il y a une recension exhaustive des publications concernant les droits de propriété des femmes en Nouvelle-France dans le chapitre 6 de mon livre, Along a River: The First French Canadian Women (University of Toronto Press, à paraître). Adoptant un point de vue divergent, les chercheuses France Parent et Geneviève Postelec, «Quand Thémis rencontre Clio : les femmes et le droit en Nouvelle-France », Cahiers de droit (Québec), 36,1 (1995): 293-318 soulignent l'étendue de la participation légale et commerciale des femmes tandis que Brun, dans Vie et mort du couple en Nouvelle-France, tend à souligner le contraire. Comme le note Brun, ce sera un progrès quand les études sur l'application de la Coutume de Paris en NouvelleFrance commenceront à examiner de plus près les changements au cours du siècle et demi d'existence de la Nouvelle-France. Cela pourrait aider à expliquer les divergences des études existantes, peut-être en révélant un parallèle avec le déclin d'une certaine tolérance envers les femmes propriétaires et commerçantes en Amérique à mesure que la vie coloniale devenait plus organisée et en quelque sorte plus capable de reproduire les pratiques de la métropole.

21. Amy Erickson, Women and Property in Early Modern England (Londres, Routledge, 1993), 233. En plus de ses pratiques moins égalitaires quant aux héritages, la Common Law accordait moins de droits aux douairières, et des droits moins sûrs, que ne le faisaient les autres codes. 
nourrir ses enfants ${ }^{22}$. D’autres étaient agressives et incorrigibles. Quand Richard Pretty, shérif d'Albany, fouilla la maison de Grietje Borsboom à Schenectady en 1680 et trouva des fourrures cachées, Grietje menaça de le scalper et tenta de le soudoyer; une autre fois, son fils et elle l'attaquèrent. Ariaentje et Geertruy Hoogeboom, d'Albany, furent condamnées, ainsi que leur mère, en de nombreuses occasions pour avoir vendu des articles tels que des breloques, de la peinture ou des couteaux. En 1676 la cour infligea des amendes à "five defendants, four of whom were women... charged with sending children to the Indian houses for the purpose of trade[...]». Deux ans plus tard, quatre des cinq accusés pour des faits similaires étaient des femmes, le shérif se plaignant de ce que «incessantly, contrary to ordinance, venture again and again either to go themselves or to send their children to the Indian houses on the hill and to trade ${ }^{23}$. . Cinq des neuf défendeurs étaient filles de bourgeois d'Albany, qui ne craignaient apparemment pas que le commerce illégal ternisse leur réputation. Pour des raisons évidentes, nous ne saurons jamais combien il y eut exactement de ces transactions illégales, mais les preuves indiquent que les délits étaient commis à répétition par des femmes de toutes classes sociales, qui impliquaient sans vergogne leurs enfants dans ces transactions.

Les négociants coloniaux suivirent les Premières Nations à la trace. De nouvelles données chiffrées sur ce groupe tendent à confirmer un degré très élevé de participation des femmes à la traite des fourrures. La traduction récente (du néerlandais à l'anglais) et l'analyse par Kees-Jan Waterman des registres de la famille Wendell d'Albany en 1695-1726 s'ajoutent désormais aux découvertes classiques de l'historienne canadienne Jean Lunn. Cela complète le portrait des Premières Nations qui apportaient des peaux à Albany pour y faire de meilleures affaires qu'avec la Compagnie française des Indes occidentales, qui détenait un monopole au nord de la frontière. Albany offrait des produits de luxe comme des huîtres, du sucre blanc, des rubans et de la dentelle, en sus des produits de base du négoce tels que wampum, rhum et lainages anglais. Passer en fraude ces denrées entre le New York et le Canada rapportait des commissions aux porteurs,

22. Voir Jaap Jacobs, New Netherland: A Dutch Colony in Seventeenth Century America (Leiden, Brill, 2005), 208-209 pour le cas de la pauvre Susanna Jans, au milieu du xvir ${ }^{\mathrm{e}}$ siècle, qui prétend échanger de l'alcool contre un castor dont elle voulait nourrir ses enfants. Jacobs livre un survol utile de la traite des fourrures en Nouvelle-Hollande (191-214).

23. Voir Dennis Sullivan, The Punishment of Crime in Colonial New York: The Dutch Experience in Albany During the Seventeenth Century (New York, Peter Lang, 1997), 167-168. 
certains ayant été retrouvés à la mission du Sault-Saint-Louis ${ }^{24}$. Quelque trois cent vingt-cinq Indiens ouvrirent des comptes avec les frères Evert, Harmonius et Hester Wendell durant la période 1695-1726, la majorité appartenant aux nations iroquoises ${ }^{25}$. Les négociantes tenaient un rôle actif dans 49,6\% des comptes. Dans ces comptes (qui mentionnent souvent une poignée de négociants issus du même groupe ou de la même famille), les femmes comptaient pour 25 à $30 \%$ des personnes faisant des transactions. Un autre livre de compte hollandais de New York, que Waterman a analysé, donne des résultats presque identiques ${ }^{26}$.

En plus de venir souvent négocier leurs pelleteries, ces femmes assumaient les mêmes responsabilités et rôles spécialisés dans le commerce que les hommes. Amener des camarades à la rencontre des marchands d'Albany et servir de caution à ces nouveaux venus quand ils achetaient des denrées en faisaient partie, tout comme emporter des marchandises de traite d'Albany pour les vendre ailleurs. Environ $10 \%$ des négociants autochtones venaient du Canada, y compris des femmes dénommées Ohonsaioenthaa, Okaajthie, Anna et Quanakaraghto. Les femmes étaient plus souvent identifiées purement et simplement comme l'épouse, la sœur ou la mère d'untel qui apparaissait ailleurs dans les comptes, ou par quelque caractéristique personnelle. Par exemple, une «sauvagesse boiteuse » descendit du Canada pour la première fois en 1697, amorçant plus d'une décennie d'échanges de peaux d'ours, de daims, d'orignaux et de martres contre des bouilloires ou des chemises. Trois ans plus tard, en dépit de son infirmité, elle engageait huit castors pour obtenir du mar-

24. Jean Lunn, «The Illegal Fur Trade out of New France, 1713-1760», Canadian Historical Association, Historical Papers (1939): 61-76.

25. Kees-Jan Waterman, dir. et trad., "To Do Justice to Him and Myself " : Evert Wendell's Fur Trade with Indians in Albany, New York, 1695-1726 (Philadelphie, American Philosophical Society, 2008), 310 p. Waterman note qu'une autre main, inconnue, dans les comptes pourrait être celle de leur mère Ariaantze.

26. Plus précisément, les femmes faisaient $24,9 \%$ des achats et $31,5 \%$ des paiements sur les comptes des deux registres hollandais de New York que Waterman a étudiés (il ne fournit pas de chiffre global, mais il affirme que les dettes n'étaient pas toutes payées, de sorte qu'on peut supposer qu'il y avait plus d'achats que de ventes et que le chiffre était plus près de 25 que de 31 ). Le second livre de compte était celui d'un négociant inconnu du comté new-yorkais d'Ulster, en 1712-1732. La preuve est renforcée par le fait que ces deux sources indépendantes révèlent des taux de participation des négociantes très similaires, également élevés, en dépit du fait que les clients des Wendell étaient surtout d'origine iroquoise tandis que ceux du comté d'Ulster étaient principalement d'origine algonquine. Une traduction abondamment annotée du livre de comptes d'Ulster est sous presse. Waterman a résumé quelques-unes de ses découvertes concernant les deux séries de comptes dans une communication inédite au congrès Omohundro, en juin 2008 à Boston, «Indian Women in the Fur Trade in Colonial New York, 1695-1732». 
chand un canot français avec lequel elle retourna au Canada ${ }^{27}$. Ayant apparemment la confiance des Wendell, elle servit de caution pour une autre négociante. En une autre occasion, une femme mohawk du Canada, vérolée, arriva pour négocier juste après Noël en 1705, apportant les "salutations du prêtre». Elle acquit des bas de grosse laine rouge et neuf barres de plomb. Avec quelques autres membres de sa famille, elle négocia avec les Wendell durant plusieurs années, échangeant des martres et des castors contre des articles tels que des bas, des couvertures et des manteaux $^{28 .}$

Axées sur une décennie ultérieure, celle des années 1750, les minutieuses investigations de Jean Lunn relient les événements mentionnés dans les plaintes des officiels canadiens au sujet de la contrebande avec ceux notés dans les registres de Robert Sanders, un marchand d'Albany. Sanders faisait affaire avec six colporteurs attitrés, dont deux femmes, Agnesse et Marie-Magdeleine. Comme les femmes dans les comptes des Wendell, elles franchissaient régulièrement pour affaires les 210 milles $(340 \mathrm{~km})$ entre Montréal et Albany, Agnesse bouclant le trajet trois fois entre mai et juillet 1753. Les canots étaient typiquement chargés de barils d'huîtres ou des cargaisons de 130 livres (60 kg) de castor. Il y avait aussi des femmes parmi les clients canadiens-français de Sanders; il expliquait dans une lettre à l'une d'elles qu'il envoyait du drap en échange de ses fourrures mais qu'il serait inutile d'ajouter de la dentelle parce que l'intermédiaire la retirerait pour la vendre à des amis ${ }^{29}$. Les Indiennes, comme les négociants hollandais, connaissaient apparemment leurs affaires et faisaient preuve d'une certaine indépendance.

\section{UN RÉSEAU DE CONTREBANDE}

Nous retrouvons quelques bourgeoises au cœur du commerce illégal entre Montréal et Albany. En 1743, le gouverneur Beauharnois et l'intendant Hocquart informèrent tous deux la Cour de France de leurs soupçons

27. K.-J. Waterman, op. cit., 133 et notes afférentes. Il ne traduit pas le terme «kno» mais explique dans le glossaire (p. 95) que c'est la façon dont les marchands hollandais écrivaient "canot».

28. Ibid., 164-165, 180 et notes afférentes. Il croit que le prêtre qui envoyait ses salutations était de la région de Montréal. Les jésuites qui travaillaient à la mission du Sault-Saint-Louis furent longtemps soupçonnés de favoriser le commerce illicite avec les Anglais (en partie pour éviter à leurs convertis les tentations plus proches de Montréal). Les tensions sur ce point culminèrent avec l'expulsion du père Jean-Baptiste Tournois en 1750. Pour la proportion de négociantes, voir: K. J. Waterman, op. cit., 48.

29. Sanders Letterbook, 11 septembre 1753. 
à l'égard des demoiselles Desauniers: Marguerite, Marie-Anne et Magdeleine. Le prudent Hocquart exprima sa frustration de n'avoir encore pu "réunir des preuves ou les prendre en flagrant délit». C'étaient des célibataires qui avaient atteint l'âge de la majorité et qui pouvaient donc faire commerce seules en toute légalité. Même si elles possédaient une grande et belle maison à Montréal, elles préféraient résider en leur magasin de l'autre côté du fleuve, à la mission du Sault-Saint-Louis. Là, elles avaient petit à petit accumulé de nombreux articles convenant à la traite des fourrures. Un employé de la Compagnie française des Indes occidentales avertit les autorités qu'elles n'apportaient jamais de pelleteries à ses bureaux, comme il était requis, alors que leurs clients autochtones auraient sûrement payé certaines de ces marchandises avec des fourrures. On leur fit commandement de fermer leur magasin, les sœurs ne refusèrent pas directement; mais pour quelque raison, elle continuèrent de vivre à la mission.

Être une femme pouvait avoir ses avantages au village de la mission. Les femmes haudénosaunies en gouvernaient l'essentiel des affaires, puisque les hommes étaient souvent partis en guerre ou pour trapper. Les Desauniers étaient réputées pour leurs œuvres charitables à l'égard des malades et des indigents, elles parlaient couramment la langue indigène et l'on disait qu'elles travaillaient avec des complices autochtones qui apportaient des fourrures de Montréal, cachées dans le genre de panier que l'on apportait au marché. Les sœurs, dont Hocquart disait qu'elles avaient la langue bien pendue ${ }^{30}$, réussissaient-elles à charmer non seulement les femmes autochtones mais aussi les hommes français? Des membres influents dans les milieux militaires, marchands et même ecclésiastiques intercédèrent pour elles auprès des autorités. Elles étaient sans reproche; elles avaient un long passé de négociantes à Montréal et au Sault, conduisant leurs affaires durant quelque quarante-quatre ans avec "probité... honneur et distinction ${ }^{31}$ ".

Cela dit, quand certains informateurs autochtones admirent en 1750 que les membres du réseau non seulement commerçaient avec les Anglais au sud, mais se rendaient dans les pays d'en-haut avec des canots chargés de contrebande ${ }^{32}$, le gouverneur général envoya des troupes pour les

30. Il les décrivit comme "fertiles en beau parler, inégalées à présenter mensonges et vérité avec une égale conviction».

31. Bibliothèque et Archives Canada (BAC) C11A, vol. 97 (10 oct. 1751).

32. BAC, C11A 1750, vol. 95, fol. 174-175. Les deux chefs de guerre du Sault-Saint-Louis fournirent des détails : trois canots chargés de marchandises anglaises avaient pris la direction de l'ouest 
évincer. Sa mort prématurée donna aux Desauniers une chance d'accabler son successeur par intérim de leurs soucis. Ne pourraient-elles point visiter la mission une dernière fois, pour une seule journée, afin de se faire rembourser les dettes contractées par leurs clients? Des mois plus tard, un nouveau gouverneur général arriva pour les trouver encore sur place. Il envoya un ordre final, et elles s'en furent enfin.

Étrangement, une pleine saison de traite ne s'était pas écoulée que le poste militaire proche du magasin fermé des Desauniers devint un centre d'activité. Il semblerait qu'une autre femme les ait remplacées. Le registre de Sanders enregistre l'envoi de contrebande à l'épouse de M. de Merceau, le commandant du fort au village de Sault-Saint-Louis, voisin des sœurs désormais expulsées. Madame de Merceau semble avoir suivi les façons de faire de plusieurs épouses de commandant de poste, qui s'adonnaient au commerce. Madeleine Bouat, la femme du commandant du fort SaintFrédéric (Crown Point, New York), travailla à rendre rentable ce poste qui rapportait peu, ouvrant un magasin aux marchandises variées, y compris de la contrebande passée par le pays iroquois et acquise en échange de ses spiritueux $^{33}$. À fort Détroit, les deux épouses successives ${ }^{34}$ du commandant Alphonse de Tonty se consacrèrent à des transactions commerciales qui suscitèrent aussi les soupçons; la seconde épouse, Marie-Anne La Marque, fut impliquée dans un commerce illégal à Montréal avant de déménager dans l'ouest. Les Desauniers n'étaient pas seules à profiter du commerce des missions et des postes militaires malgré les rumeurs, les rivaux et les officiels rapaces.

\section{DES NÉGOCIANTES DE PREMIER PLAN}

Il y avait aussi des négociantes qui jouissaient d'une respectabilité sans faille, des femmes d'élite qui œuvraient des côtes du Labrador jusqu'au lac Supérieur. Il y avait des détaillantes d'envergure. Deux d'entre elles

au cours des cinq derniers jours sous le commandement d'Agouirache.

33. Cela fit scandale au fort, où son monopole était garanti par l'autorité de son mari. Madeleine Bouat (madame Lusignan) avait de l'expérience dans les affaires; les registres notariaux de Montréal notent qu'elle était procuratrice financière pour le couple et plus tard pour son fils. Son commerce au fort fut remarqué par Louis Franquet, Voyages et Mémoires sur le Canada (Montréal, Éditions Élysée, 1974), 67. Merceau s'écrivait aussi Mousseaux.

34. BAC, C11A, 1707, vol. 26, fol. 176-219, 1729, vol. 120, fol. 243-246, 1731, vol. 54, fol. 282285, 1753, vol. 99, fol. 25-90; "Tonty, Alphonse de», DCB II. Il y a de nombreuses références à madame La Pipardière dans C11A 1715-17 (vol. 35-38 et 123); 1717, vol. 38, fol. 109-116 fait allusion à son voyage pour rejoindre Tonty après que son mariage fut approuvé. Pour les accusations de traite des fourrures du fait de l'épouse, voir 1707, vol. 26, fol. 176-219, 1708, vol. 29, fol. 140-143, 1717, vol. 37, fol. 140-143, 1717, vol. 38, fol. 119-120v. 
avaient épousé à l'adolescence des hommes plus âgés et se montrèrent plus entreprenantes en affaires que leurs maris. Après que Louise Chartier de Lotbinière, fille d'un officiel éminent, eut épousé Louis Denys de la Ronde, elle reçut procuration pour administrer les affaires commerciales de son mari. Après une vaine tentative de développer une mine de cuivre près du lac Supérieur, il mourut lourdement endetté. Le fils reprit le commandement de son père au poste de Chagouamigon (dans le Wisconsin actuel), tandis que la mère s'en fut à Versailles et obtint de conserver le poste dans la famille ${ }^{35}$. Marie Louise Denys Laronde Delatrinité demanda la séparation de biens d'avec son mari, Pierre D’Ailleboust Desmusseaux D’Argenteuil, au motif que leurs affaires étaient mal gérées. Ayant survécu plus de trois décennies à son mari, la veuve d'Argenteuil amassa des avoirs de 46000 livres. Elle mena une bataille victorieuse contre les Sulpiciens pour le contrôle de la prospère seigneurie d'Argenteuil, à l'ouest de l'île de Montréal ${ }^{36}$. Après avoir retenu un voyageur pour lui rapporter des pelleteries de Michilimackinac, elle se lança dans le tannage. Deux autres femmes d'affaires bien connues, de noble famille, Agathe de Saint-Père et Louise de Ramezay, s'associèrent aussi à des tanneries, profitant de la rentabilité accrue des peaux au XviII ${ }^{\mathrm{e}}$ siècle. Nous ne connaissons pas le volume du commerce de Madeleine Roybon d'Allone, qui pourrait avoir été du petit nombre de filles $d u$ roi issues de la noblesse. Elle ne se maria pas, mais prit une concession territoriale près du fort Cataraqui, subit la captivité chez les Iroquois, puis, libérée, pratiqua le négoce de spiritueux et d'autres articles. Elle demeura longtemps en affaires, écrivit des pétitions au nom d'un groupe de négociants du lac Ontario et commerça des années 1670 jusqu’à 1717 au moins $^{37}$.

D’autres furent actives sur la côte du Labrador. En ce qui concerne Marie-Charlotte Charest, épouse du sieur de Courtemanche, «[i]l semble

35 BAnQ-M Pinguet de Vaucour, J-N, 29 avril et 4 mai, procurations ; 21 oct. 1734, obligation; 5 oct. 1746, inventaire, 17 oct. 1746, renonciation; BAC, C11A, 1747, vol. 87, fol. 171-172v; 1748, vol. 91, fol. 24-25v; 1751, vol. 97, fol. 201v.

36. Les activités de Marie Louise Denys de la Ronde sont traitées dans Lorraine Gadoury, Une famille noble en Nouvelle-France : les D’Ailleboust, mémoire de maîtrise, Université de Montréal, 1982, voir surtout 74-75, 107-115 ; et Louise Tremblay, La politique missionnaire des Sulpiciens au XVII et début du XvIIr siècle, mémoire de maîtrise, Université de Montréal, 1981, surtout 144-148; C11A, 1717, vol. 39, fol. 217-18, 1731, vol. 56, fol. 97-9; "Ailleboust D’Argenteuil, Pierre d'», DCB II. BAnQ-M, Adhémar, J.-B., 7 mai 1717, engagement; 21 sept. 1723, convention et société. Une très généreuse dot pour le mariage de sa fille en 1732 suggère que ses entreprises se portaient bien ainsi que les comptes présentés par Gadoury.

37. Pour un compte rendu, voir «Roybon d'Allone, Madeleine de », DCB II. 
que Mme de Courtemanche fut une femme forte qui avait son mot à dire dans les affaires du poste ${ }^{38} »$. Elle parlementait avec les Inuit et avec les officiels à la Cour de France au nom de la compagnie, survécut à une tentative d'assassinat et prit des mesures pour faire entrer ses filles dans les affaires. De même, Marie-Anne Barbel, en tant que veuve avec cinq enfants, étendit le commerce familial de fourrures et d'huile d'animaux marins. Vingt-quatre hommes travaillaient pour la «Veuve Fornel et Compagnie», société qu'elle forma en association avec les marchands Havy et Lefevre ${ }^{39}$.

Les opérations s'étendaient souvent sur de grandes distances. Charlotte Françoise Juchereau de St-Denys et Thérèse Migeon consentaient des prêts aux marchands de fourrures et s'associèrent à des regroupements de négociants qui rivalisaient pour obtenir l'agrément de Versailles ${ }^{40}$. Mesdames Baby et St-Anges Charly étaient des membres actifs de familles marchandes qui fournissaient le commerce de l'Ouest. L'historienne Kathryn Young a compté jusqu'à trente femmes dont les entreprises touchaient d'une façon ou d'une autre au commerce des fourrures, y compris deux expéditrices, Marie-Anne Busquet et sa belle-sœur (Mme Catignon et Mlle Catignon), qui travaillèrent une décennie durant à Québec comme agents coloniaux d'une firme de La Rochelle ${ }^{41}$. Marguerite Bouat, veuve Pascaud, géra avec succès la plus importante compagnie de La Rochelle faisant commerce avec le Canada, durant une décennie après la mort de son mari en 1717; elle inspira suffisamment confiance au gouverneur Vaudreuil pour qu'il recommande qu'on la nomme commissionnaire préposée aux achats pour la colonie à La Rochelle ${ }^{42}$. Depuis le fort Niagara, madame Celeron envoyait du grain en aval du Saint-Laurent. Les registres comptables de Havy et Lefebvre, agents de Rouen pour le

38. “Acoutsina», DCB II.

39. BAC, C11A, 1750. vol. 96, fol. 104-108 donne le propre compte rendu par la veuve Fornel de son négoce de peaux et d'huile d'animaux marins. Sur ses activités, voir L. Plamondon, "Une femme d'affaires en Nouvelle-France: Marie-Anne Barbel, veuve Fornel», loc. cit., 165-185.

40. "Juchereau de Saint Denys, Charles de», DCB II; BAC, C11A, 1702, vol. 36, $411 \mathrm{ff}$.

41. Kathryn Young, Kin, Commerce, Community: Merchants in the Port of Québec, 1717-1745 (New York, Peter Lang, 1995), 22.

42. BAC, C11A, 1717, vol. 38, fol. 133-137v; 1723, vol. 45, fol. 121-123v; Dale Miquelon, New France 1701-1744, "A Supplement to Europe" (Toronto, McClelland and Stewart, 1987), 81, 127. Les registres notariaux conservent une série de transactions y compris des procurations donnant pouvoir à l'épouse de Pascaud d'agir légalement en son nom en son absence dès 1697 . Voir aussi BAnQ-M, Adhémar, A, 3 oct. 1697, procuration. Sur Mme Pascaud, voir également John Bosher, Men and Ships in the Canada Trade, 1660-1760: A Biographical Dictionary (Ottawa, Ministry of Supply and Services, 1992), 101-103. 
Canada dans la traite des fourrures, notent aussi des comptes d'expédition avec mesdames Guy, Brunet et Moquin, ainsi que mesdemoiselles Auger, de Joncaire et Texier ${ }^{43}$.

\section{DES NÉGOCIANTES PLUS MODESTES}

Dans la région des Grands Lacs, d'innombrables femmes d'origine autochtone piégeaient et traitaient. Bien qu'elles soient difficiles à retracer, elles ont commencé à émerger dans les études de Susan Sleeper Smith, Bruce White et d'autres chercheurs ${ }^{44}$. Dans les postes français et dans les alentours, les registres révèlent de nombreuses transactions par des femmes aux noms français, beaucoup étant probablement métisses ou des conjointes autochtones de Canadiens. À Détroit, elles fournissaient des biens et services à la mission jésuite ou à la Couronne. Madame Goyau commença à faire la blanchisserie et la boulange pour la mission chez les Hurons en 1743, au salaire annuel de 100 livres. Elle réceptionnait aussi les marchandises de traite comme les fusils et leur poudre, les chemises, les couvertures, l'eau-de-vie et les pois. Madame Pilette moulait du grain pour la mission. Mesdames Mallet et St-Martin vendaient de l'eau-de-vie, des bas et des objets divers à la mission tandis que madame Martin, avec mesdames Cuillerier et L'Eil Éraillé, achetaient, entre autres biens, de la poudre, des munitions, des couteaux, des perles de verre, du vermillon, des chemises et des chaussures. Le missionnaire avait retenu «la femme de Gambille" pour vendre à commission quantités de porcelaines, de couvertures, des mitasses ${ }^{45}$, des chemises de diverses tailles et plusieurs livres de vermillon, cependant que "la femme de Caron» échangeait des clous, des lanières en daim et des perles de porcelaine. Mademoiselle

43. K. Young, "..."sauf les perils et fortunes de la mer": Merchant Women in New France and the French Transatlantic Trade, 1713-1746", loc. cit., 39-40.

44. Les découvertes de Waterman sur la très forte proportion de négociantes haudénosaunies à Albany sont examinées ci-dessus. Pour les négociants des pays d'en haut, voir Susan Sleeper Smith, «Women, Kin and Catholicism: New Perspectives on the Fur Trade», et Bruce M. White, "The Woman Who Married a Beaver: Trade Patterns and Gender Roles in the Ojibwa Fur Trade», les deux dans Mary-Ellen Kelm et Lorna Townsend, dir., In the Days of Our Grandmothers : A Reader in Aboriginal Women's History in Canada (Toronto, University of Toronto Press, 2006) : 26-55, 56-92. Pehr Kalm observa au passage cette participation, sur une route près de Niagara où il rencontra un grand nombre d'Indiens des deux sexes qui transportaient leurs peaux et d'autres denrées à Québec. Voir A. Benson, Peter Kalm's Travels in North America (New York, Dover, 1937), 696. Le gouverneur Beauharnois en eut un autre aperçu, relatant que les chefs des Outaouais lui avaient promis qu'ils n'iraient pas à Chouaguen et rapportant son déplaisir quand leurs épouses y allèrent à leur place. BAC, C11A, 1742, vol. 76, fol. 225-230v.

45. Canadianisme : guêtre en peau de chevreuil ou en drap, ornée de dessins ou de poil d'orignal. $(\mathrm{NdT})$ 
Royale et madame Skotache faisaient le commerce de couteaux, de briquets d'acier, de perles et de vermillon, et madame Delile de viande ${ }^{46}$.

Dans les villes le long du Saint-Laurent, la traite des fourrures générait aussi des profits. Du côté des acheteurs, il y avait la veuve Monfort, qui était venue à Québec en 1742 du poste de la rivière Saint-Joseph (dans le Michigan actuel) afin de se procurer de la toile de traite, des fusils et des plombs, des bouilloires et des haches pour une valeur de quelque onze mille livres ${ }^{47}$. Cette commande considérable contrastait avec les innombrables transactions plus humbles dans les villes laurentiennes, la plupart n'ayant probablement jamais été enregistrées. Du côté des encaisseurs, il y avait Marguerite Launay et plusieurs autres citadines compensées par la Couronne pour avoir fourni aux douzaines de visiteurs des Premières Nations du logement, de la nourriture ou du bois de chauffage, tandis que la veuve Laprairie était compensée pour avoir opéré un cabaret qui fournissait de la bière à ces alliés ${ }^{48}$. Les petits montants associés à nombre de ces transactions mettent en évidence un point important: le petit commerce était une activité quotidienne pour les gens ordinaires.

Si les fondatrices d'entreprises étaient une minorité, les petites commerçantes semblables à celles qui apportaient leurs produits et leur artisanat au marché local se retrouvaient partout. Dans les années 1740, le voyageur suédois Pehr Kalm nota que les Canadiennes (contrairement aux femmes qu'il avait croisées dans les sociétés plus aisées de l'Angleterre et de l'Amérique) se consacraient à la production et aux échanges économiques. Il remarqua combien "women in Canada... do not spare themselves, especially among the common people, who are always in the fields, meadows, stables, etc., and do not dislike any work whatsoever...» Kalm nota que «The common people in the country seem to be very poor. They are content with meals of dry bread and water, bringing all other provisions, such as butter, cheese, meat, poultry, eggs, etc to town, in order to get money for them. " La plupart de ces denrées étaient d'habitude produites par des femmes. Bien que ses récits de voyage et ceux d'autres observations de l'Angleterre et de l'Amérique aient noté des fermières qui commençaient à se retirer de certains aspects de la production et de

46. Ces échanges sont enregistrés dans le Journal des Jésuites (1710-1755) et cités dans: Katherine Lawn et Claudio Salvucci, Women in New France: Extracts from the Jesuit Relations (Bristol, PA, Evolution Publishing, 2005), 288-290.

47. La veuve est signalée dans D. Miquelon, op. cit., 132-133, 153.

48. Voir par exemple BAC, C11A, 1716, vol. 36, fol. 389-92; 1717, vol. 37, 363-364v, 426-427v, 439-443; 1720, vol. 42: 156-158. 
la mise en marché, Kalm découvrit au Canada que non seulement des femmes travaillaient aux champs, mais que «daughters of all ranks, without exception, go to market $^{49}{ }^{\prime}$.

\section{LES FEMMES EN TANT QUE FOURNISSEURS EN TEMPS DE GUERRE}

L'affrontement des empires rivaux de la France et de l'Angleterre au XviII ${ }^{\mathrm{e}}$ siècle entraîna des investissements militaires massifs de la France en Nouvelle-France, ces dépenses connaissant une escalade ininterrompue depuis le temps de la guerre de la Succession d'Autriche de 1740-1748 jusqu'à la conquête de la colonie par la Grande-Bretagne en $1760^{50}$. Quand les officiers et les hommes furent appelés au front en 1744, le marchand Pierre Guy rapporta de nombreux transferts aux épouses de pouvoirs de mandataire d'affaires ${ }^{51}$. Il semble plausible que certaines d'entre elles se révélèrent des agents plutôt passifs pour les entreprises de leur mari. Mais beaucoup de femmes n'avaient pas de mari, ou ne l'avaient pas eu à leurs côtés depuis des années, et elles étaient clairement les maîtresses de leurs propres entreprises.

Cette partie s'appuiera principalement sur les comptes exceptionnellement détaillés que l'intendant Gilles Hocquart tint des dépenses du gouvernement français au Canada durant les années 1740, et aussi sur quelques autres sources contemporaines. Ils fournissent un aperçu peu exploité de la participation des femmes au commerce.

Les données tirées des comptes de la Couronne pour la Nouvelle-France confirment les analyses de l'historien Peter Earle pour Londres au début

49. Les remarques de Kalm sur les Canadiennes se trouvent dans A. Benson, Peter Kalm's Travels in North America, (New York, Dover, 1937), 403, 417, 479, 525-526. En 1679, un rapport officiel observa aussi femmes et enfants affectés aux travaux de la ferme et au soin du cheptel, ce qui fut attribué à l'absence de cinq ou six cents coureurs des bois. BAC, C11A, 1679, vol. 5, fol. 32-70. Sur la situation anglaise, voir Robert Shoemaker, Gender in English Society 1650-1850: The Emergence of Separate Spheres? (Edimbourg, Longman, 1998), 156-159, 174, 189. Shoemaker cite une observation anglaise de 1800 selon laquelle une fermière défaillerait à l'idée d'aller au marché, comme sa mère et sa grand-mère, avec un panier de beurre, de porc, de cochons de lait ou de volailles sous son bras. Voir aussi Joseph Lucas, trad., Kalm's Account of his visit to England on his way to America in 1748 (Londres, Macmillan, 1892), 80-95, 327-328.

50. Historical Atlas of Canada (Toronto, University of Toronto Press, 1987), I, planche 48. Selon une estimation, les dépenses augmentèrent d'environ un demi-million de livres au début des années 1740 à quelque 2,5 millions annuellement en 1744-1748 («Hocquart, Gilles», DCB IV); Selon une autre estimation elles doublèrent ou triplèrent. (Catherine Desbarats, «Les deniers du Roi dans l'économie canadienne du XVIII ${ }^{e}$ siècle», dans Sylvie Depatie et al., dir, Vingt ans après Habitants et marchands: Lectures de l'histoire des XVII et XVIII siècles canadiens (Montréal, McGillQueen’s University Press, 1998), 194.

51. BAC, Coll. Baby, Correspondance, II, Havy et Lefebvre à Madame Guy; 3 août 1745, 664665. 
du XviII ${ }^{\mathrm{e}}$ siècle où, dans la plupart des classes sociales, il n'y avait pas grande objection au travail des femmes, bien au contraire. Selon son étude, de nombreuses femmes déclaraient un revenu de sources sans rapport avec l'activité de leur mari. Dans les économies pré-industrielles, il n'y avait rien d'exceptionnel à ce que des femmes décident de participer à l'économie marchande, encore que pour la plupart il devait s'agir d'une activité informelle ou occasionnelle, non de l'occupation à plein temps que sous-entend le terme "femme d'affaires». Ce n'était pas une situation particulièrement «favorisée», mais une nécessité du quotidien dans une colonie où les difficultés économiques n'étaient pas rares même au sein des classes privilégiées. Explorons les multiples voies par lesquelles les femmes tiraient un revenu des coffres du gouvernement durant les dernières décennies du Régime français.

\section{FOURNISSEUSES DE CHANTIERS NAVALS}

D'énormes troncs furent abattus et sciés pour servir aux chantiers navals et aux fortifications de Québec quand les activités militaires prirent de l'expansion durant les années 1740. La veuve Baron de Lupien, mère de dix enfants, pourrait sans doute être identifiée comme une "déléguée du mari», car elle n'entra en affaires que pour une année, le temps d'exécuter le contrat de 34000 livres conclu par son mari avant sa mort et l'engageant à fournir du chêne du lac Champlain et autres bois pour les chantiers navals de Québec ${ }^{52}$. D’autres, cependant, étaient plus autonomes. MarieAnne Hazeur, une orpheline formée par les ursulines, avait vingt ans quand elle épousa Michel Sarrazin, qui en avait cinquante-trois. La mort de ce médecin respecté mais mal payé laissa sa famille dans la gêne. Durant son long veuvage, Marie-Anne et sa fille vendirent des épinettes de leur propriété de Sainte-Foy pour la construction de navires de la Couronne comme le Castor et la Martre. Louise de Ramezay, fille célibataire de feu le gouverneur de Montréal, y participait également, en expédiant des mâts et des planches de chêne et de pin provenant de sa seigneurie de la vallée du Richelieu pendant les dernières décennies du Régime français ${ }^{53}$.

Il y avait d'autres joueuses, grandes et petites; certaines avaient des maris, d'autres non. En plus de veiller aux affaires de son mari, posté dans l'ouest, Marguerite Saint-Ours Deschaillons recevait 1800 livres de la

52. BAC, C11A, 1744, vol. 81, fol. 277-289; «Lupien, dit Baron, Pierre», DCB III.

53. BAC, C11A, 1741, vol. 75, fol. 307-308v; 1742, vol. 78, fol. 256-269; 1745, vol. 84, fol. 71v; «Sarrazin, Michel», DCB II. 
Couronne pour du bois et des matériaux de construction, et elle fournissait des rations à 4 navires différents en 1747. D’autres profitèrent aussi des projets de construction en temps de guerre. Madame Duberon empocha 767 livres pour avoir fourni du bois de construction de navires, et durant les quelques années suivantes, quelques milliers de plus en rapport avec la fortification de Québec et d'autres dépenses de guerre. Madame Desrusseaux était payée pour le bois de ses terres transformé en cages utilisées pour la construction de la flûte le Caribou. La demoiselle Sabrevois et une autre dame furent également payées pour du bois mis en trains. En 1747, mademoiselle Bayeul fut compensée au chapitre des achats de bois et des constructions. En plus des grosses livraisons, il y en avait de petites, les dames Portneuf, Youville, Marguerite Dupuy et la veuve Françoise Lamoureux fournissant des chargements de bois évalués entre cinquante et sept cents livres ${ }^{54}$.

Le nombre et la variété des fournisseurs sont étonnants. Pour l'année 1746, 598 hommes et femmes sont recensés comme fournisseurs de vivres, munitions et marchandises, un groupe que Catherine Desbarats caractérise comme "une multitude de modestes fournisseurs" dans une colonie qui n'engendrait pas de grands capitalistes seigneuriaux ou marchands comparables à ceux de la France ${ }^{55}$. La traite des fourrures déclina en temps de guerre, de sorte que ses fournisseurs habituels se tournèrent vers la Couronne. Toutes sortes de marchandises - bois, fer, clous, pots d'eau, choux, oignons - parvenaient au chantier naval gouvernemental, apportées par les veuves Lajus, Lagrois, Julienne Larche (dont le mari avait été marchand de viande) ainsi que par la veuve Pommereau et sa sœur (une paire qui faisait des profits supplémentaires en louant une chambre à un employé de la Couronne). Les constructeurs de navires ou leurs équipages étaient aussi logés par la veuve Lefebvre, madame Girard, madame Gouze et madame Duplessis, cette dernière louant deux maisons à l'équipage de La Martre à cinquante livres par mois. Madame Langloiserie, Marie Charlotte Palin, Marie Paquet et Marie Pement complétaient la liste des fournisseuses ${ }^{56}$.

54. BAC, C11A, 1732, vol. 113, fol. 470 (Dupuy); 1740, vol. 114, fol. 287v (Portneuf et Youville); 1742, vol. 78, fol. 243-254; vol. 79, fol. 327-342v; (Lamoureux) 1744, vol. 84, fol. 72v et 1747, vol. 89, fol. 120-122v et vol. 115, fol. 171v, vol. 92, fol. 202-219v; (Sabrevois) 1743, vol. 80, fol. 62ff.

55. C. Desbarats, loc. cit., 201-203.

56. BAC, C11A, 1742, vol. 78, fol. 137-141 et 173-224v; 1743, vol. 80, fol. 186-187v, 191-192v et 217-218v; 1744, vol. 82, fol. 29-53; 1745, vol. 83, fol. 195-250v; 1746, vol. 85, fol. 369-374; 1747, vol. 89 , fol. $120-122 \mathrm{v}$ et vol. 115 , fol. $171 \mathrm{v}$; 1749, vol. 93, fol. 321-322, 392-398v; «Pommereau, J.-B. », DCB III. 
Ces femmes pouvaient recevoir des lettres de change, dépassant parfois les 2000 livres, pour des services variés (parfois non précisés) en rapport avec la construction navale, dont la location d'une chambre ou d'un poêle pour réchauffer le bureau de construction, ou l'équipement d'un navire (mesdames Berthier, des Anges, de La Richardière et mesdemoiselles de Sabrevois et Gouze étaient du nombre). Dans le Domaine royal, la veuve Marie-Anne Barbel, chef d'entreprise, perçut 2000 livres pour la construction de vaisseaux et procura à la Couronne des matériaux de construction et des maisons de location. Les veuves Cureux et Rampeau reçurent aussi des paiements en relation avec les vaisseaux à quai dans le Domaine royal, en Acadie ou à Rochefort. Madame Renaud d'Avène des Méloizes, une noble veuve de la famille Lotbinière, était également du nombre ; l'entreprise de tuiles de toiture de son mari avait avorté, mais leur fille devait relever la fortune familiale en devenant la maîtresse de l'intendant Bigot. À un niveau plus humble, Marie Madeleine Duret, Marie Cluzeau et madame Jean-Pierre furent payées pour des «façons d'ouvrages et blanchissage», ce qui incluait les 21 paires de draps et 30 couvre-lits blanchis par la dernière nommée, pour 33 livres. Marie La Garenne était couturière, peut-être faisait-elle des voiles, des couvre-lits ou des vêtements pour les ouvriers et les matelots. La veuve Raymond fit plusieurs livraisons, incluant 14 pièces de cordage blanc ou de gréement pour le SaintLaurent $^{57}$. Marie Paquet, Marie Pement et la veuve Julienne Larche soignaient les ouvriers envoyés à l'Hôtel-Dieu, tandis que madame de Villeray surveillait un hôpital de campagne installé pour eux dans une maison privée.

En une autre occasion, même de jeunes femmes qui vivaient à l'ouest de la ville portuaire furent payées pour construire des embarcations militaires. Dans les années 1750, l’ingénieur français Louis Franquet visita un chantier naval à Trois-Rivières dont on disait qu'il produisait les meilleurs canots en écorce de bouleau de la colonie. C'étaient de robustes transports de troupes et de fournitures militaires destinées aux pays d'en-haut. Il rapporta: «On y travaillait un de huit places; il était de 33 pieds de longueur, cinq de largeur $[\ldots]$ du prix de 300 livres [...] ils sont destinés pour les voyages des pays d'en

57. BAC, C11A, 1741, vol. 76, fol. 105 ; 1742, vol. 78, fol. 173-191, 226-241v, 256-269; 1743, vol. 80, fol. 56-62, 186-187v, 217-219; 1745, vol. 83, fol. 195-250v; 1746, vol. 85, fol. 369-374; 1747, vol. 88 , fol. $93-107 \mathrm{v}$ et $117-131$ et vol. 89 , fol. $77 \mathrm{v}-79$; 1748 , vol. 92 , fol. 153-157, 160-169 et 224-226v et vol. 93, fol. 392-398v. Les 14 pièces de cordage de Raymond étaient dans ses provisions de maijuin pour la Saint-Laurent, qui valaient en tout 2478 livres. Sur Renaud, voir aussi «Renaud d'Avène des Méloizes, Nicolas-Marie », DCB III. 
haut, tant a porter les troupes que les vivres et marchandises $[\ldots]$ ce sont des femmes et des filles qui les travaillent ${ }^{58} \ldots$ ”

Franquet ne nous dit pas si ces filles et femmes qui construisaient les gros canots étaient des Autochtones, des Canadiennes ou quelque combinaison des deux - mais en façonnant la coque en écorce des canots, elles employaient une technique indigène, que les maris n'auraient pas supervisée normalement.

\section{NOURRIR LA MACHINE DE GUERRE}

Les femmes de tout rang et de tout statut conjugal devinrent des rouages de la machine de guerre qui se mettait en place de la colonie jusque dans l'arrière-pays. En effet, l'intendant Gilles Hocquart pensait qu'il devait autoriser non seulement les officiers pauvres mais aussi leurs veuves à se livrer au petit commerce relié à l'approvisionnement des postes en nourriture en un pays qui avait besoin de toute l'aide possible. Cela devint une politique de la Couronne de favoriser les veuves et les épouses des officiers, tant pour remonter leur moral que pour compenser des soldes trop basses pour entretenir les familles des officiers d'une manière jugée appropriée $^{59}$. Dans les comptes de la Couronne des années 1740 figurent beaucoup de ces épouses, filles et veuves relativement à la nourriture et aux munitions livrées à l'entrepôt de Québec à l'occasion de la guerre, aux fournitures pour les fortifications en cours de construction, ou aux vivres et autres denrées envoyées aux alliés amérindiens à l'intérieur des terres. Des douzaines de femmes reçurent des montants allant d'un maigre 124 livres jusqu’à 7 000. Les bénéficiaires comprenaient des célibataires: mesdemoiselles Beyeul, Manon Desauniers, Lestage et de Ramezay; des femmes mariées: mesdames Berthier, Cailleteau, de Cerry, de Lino, des Plaines, Leuron, Lusignan, Nicolet, et Tapin; et des veuves: Baby, Delaurier, Lafontaine, Petel et Raymond. Une veuve, madame Philibert, qui fournissait du pain à fort prix aux troupes coloniales et aux marins, était payée pour se taire parce qu'un noble du clan Repentigny avait tué son époux durant une dispute à propos d'un loyer. Au village haudénosauni au sud de Montréal, les sœurs Desauniers, célibataires, que nous avons rencontrées au centre d'un réseau de contrebande, se remplissaient aussi les poches avec les subsides de la Couronne pour l'approvisionne-

58. L. Franquet, op. cit., 17.

59. Par exemple, voir la discussion des privations des familles d'officiers dans les postes où les revenus ne couvraient pas les onéreuses dépenses pour le quotidien. BAC, C11A, 1748, fol. 148151. 
ment des guerriers de passage ${ }^{60}$. Plusieurs autres femmes étaient aussi payées pour fournir de la nourriture.

\section{FAIRE DES VÊTEMENTS ET DES TENTES}

La machine de guerre avait aussi besoin de travaux d'aiguille, pour compléter les uniformes envoyés de France ainsi que des articles comme des tentes de campagne et des vêtements spécialisés pour les Français et leurs alliés des Premières Nations. Les métiers d'aiguille ne cessèrent de prendre de l'importance tout au long de l'histoire de la Nouvelle-France. En novembre 1685 , le gouverneur Denonville notait, à propos des filles et femmes canadiennes, que "les menus ouvrages de capots et de chemises de traite les occupent un peu pendant l'hiver ${ }^{61}$ ». (Les tuques tricotées rapportaient aussi.) Cinquante ans plus tard, Pehr Kalm observait que les jeunes femmes de Montréal cousaient sans cesse quand elle n'étaient pas occupées à d'autres tâches. À Montréal, une couturière, Geneviève LaTerreur, récolta près de 1200 livres durant les 4 derniers mois de 1745 et Marie Coupin un dixième de cette somme; Marie Daneau cousit 13 tentes pour 156 livres. Marianne Métivier exécuta 57 capots et vingt-cinq capots galonnés pour 160 livres. Josette Métivier et Thérèse Dyon cousirent des culottes pour les troupes et d'autres articles pour les magasins de la Couronne ${ }^{62}$. Dame Poulier cousit 139 chemises de traite pour 52 livres. Dame DeNoyelles gagna 230 livres en procurant à 7 soldats autant de courtes vestes et de culottes, de paires de bas, de chapeaux, 14 chemises et cravates et 13 paires de souliers ${ }^{63}$. Madame Benoist, épouse du commandant d'Oka, supervisait une opération à Montréal qui produisait des chemises et des jupons pour les Premières Nations amies ${ }^{64}$. À l'Hôpital Général de Montréal, les directrices

60. Sur les Desauniers, voir ANC, C11A, 1750, vol. 97, fol. 384 et Lunn, «The Illegal Fur Trade out of New France, 1713-1760", loc. cit. Les autres sont citées dans 1744, vol. 82, fol. 254-255v; 1745, vol. 84, fol. 200-205 et vol. 115 , fol. 162 et 192 ; 1746, vol. 117, fol. 49-74; 1747, vol. 88, fol. $248-254 \mathrm{v}$ et vol. 89 , fol. $77 \mathrm{v}-79$ et $103 \mathrm{v}-107 \mathrm{v}$; 1748, vol. 92 , fol. 127-130v, 160-169, 236-255 et 256-271; aussi: vol. 117, fol. 68-74 et 95-116. Voir aussi R. Boyer, Les crimes et les châtiments au Canada français $d u 17^{\mathrm{e}}$ au $20^{\mathrm{e}}$ siècles (Montréal, Cercle du livre de France, 1966), 114.

61. Cité dans J. N. Fauteaux, Essai sur l'industrie au Canada sous le Régime français (Québec, L.-A. Proulx, 1927), 459.

62. BAC, C11A, 1745, vol. 115, fol. 159-162. Suzanne Francour et Marie Copin (et probablement bien d'autres) cousaient et blanchissaient pour les entrepôts de la Couronne bien avant que la guerre n'éclate en terre américaine en 1744.

63. BAC, C11A, 1745, vol. 115, fol. 187 (Poulier et LaNoyelles). Dans un autre secteur, la «dénommée Duval» et d'autres furent payées pour laver les vêtements des soldats malades.

64. Hilda Neatby, Québec, The Revolutionary Age (Toronto, McClelland et Stewart, 1966), 72-73, fait référence à cette activité peu après la Conquête; Franquet rencontra Madame Benoist en 1752-1753, Voyages et Mémoires sur le Canada, op. cit., 150. Sur les femmes qui faisaient des vêtements 
dévouées, célibataires ou veuves, supervisaient la couture des tentes et des uniformes militaires par les pensionnaires veuves, abandonnées ou orphelines confiées à leurs soins. De toute évidence, les Canadiennes contribuèrent une part des milliers de vêtements militaires ou de traite conservés dans les entrepôts de la Couronne.

\section{APPROVISIONNER LES ALLIÉS AUTOCHTONES}

Beaucoup de femmes aidèrent à ravitailler les alliés indigènes dont dépendaient les fortunes militaires françaises. Marie Louise Lefaivre donna des services non précisés dans le pays d'en-haut à l'occasion d'un déplacement d'Autochtones ${ }^{65}$. Madame Barrois gagna de l'argent en fabriquant des capots ordinaires et de fantaisie, des mitaines, des capuchons, des chaussures, des chemises de traite, des sacs de voyage et des tentes qui étaient commandées par l'officier Baron de Longueuil pour divers chefs et tribus qui se rendirent à Montréal ou au fort Saint-Joseph en temps de guerre ${ }^{66}$. Une activité similaire fut enregistrée à un autre poste de traite, Michilimackinac. Manon Lavoine, veuve Chevalier, nourrit les Autochtones qui avaient accompagné l'officier français LaCorne depuis Montréal et fournit aussi un canot de 140 livres et un cheval pour un festin. Marie Laplante Bourassa procura 100 livres de graisse que le commandant LaCorne avait commandées et logea un émissaire français auprès des Autochtones à un moment tendu. La Demoiselle Blondeau reçut 36 livres pour ce qui devait être un capot des plus soignés, sa contribution aux efforts diplomatiques français ${ }^{67}$. Au fort Saint-Joseph, Marie-Madeleine de Colon de Villiers remit à M. Debreuil une facture de 655 livres qui donne une idée de l'étendue de ses affaires: 4 sacs de blé, 30 pièces de viande, de l'eau-de-vie, du pain, du tabac et du bois de chauffage, ainsi que des peaux de daim, des chemises de traite pour homme, de la toile et des couvertures à trois points. Au fort des Miamis, Marie-Louise Lefaivre les

pour la traite, voir aussi Louise Dechêne, Habitants et marchands de Montréal au XvIr siècle (Paris, Plon, 1974), 151-153, 187, 391. On commença aussi à tisser à l'Hôpital-Général de Montréal au début du XviII siècle selon BAC, C11A, 1706, vol. 25, fol. 261-264.

65. BAC, C11A, 1746-7, vol. 117, fol. 435-443, 454v et 459-462; 1748, vol. 118, fol. 178-185.

66. BAC, C11A, (Senneville) 1755, vol. 119, fol. 278-284 ; (Mme Barrois) 1749, vol. 118, fol. 378379 (un registre fascinant qui montre à quel point Détroit était une plaque tournante pour nombre de différentes tribus nommées dans le document, instructif aussi quant aux types et coûts des objets de traite). À un autre poste, Marie Louise Lefaivre fournit au commandant Douville de la farine pour les Miamis. Voir BAC, C11A, 1747, vol. 118, fol. 143.

67. BAC, C11A, 1746, vol. 118, fol. 30-31 (Laplante Bourassa); 1747, vol. 117, fol. 144-146, 338, 363-364, 383-384 ; 443, 459-462 ; 1748, vol. 118, fol. 103, 143, 178-185 (Manon Lavoine); 1749-1750, vol. 119, fol. 184, 284 (Blondeau). 
approvisionnait, ainsi que les Kickapous et d'autres alliés, en vue d'un voyage à Montréal ${ }^{68}$.

Les négociantes du Saint-Laurent assuraient aussi des livraisons dans les pays d'en haut. La veuve Julienne Larche, par exemple, qui avait aidé à équiper les vaisseaux Rubis et Caribou à Québec en 1741-1744, fournissait en 1745 du bétail, des canots et des raquettes au commandant du fort Saint-Joseph. Madame Benoist, comme nous l'avons déjà vu, approvisionnait la traite de vêtements confectionnés à Montréal. Avec Mariane Lafayette et la veuve Magnon, elle envoyait des biens tels des porcelaines, des insignes militaires, des bouilloires de cuivre rouge et du vermillon ainsi que du verre et, pour une raison inconnue, des cornets de papier. Les variétés d'étoffe (d'une valeur de 1200 livres) fournies par la veuve L’Esperance allaient peut-être aussi à la traite de l'ouest ${ }^{69}$.

\section{LE TRAVAIL DANS LES FORTS}

Un autre groupe de femmes assurait le bon fonctionnement des forts. Même dans les années 1740, quand on fit des efforts pour réduire le nombre des femmes restant dans les postes, l'intendant Hocquart nota que certaines étaient indispensables, comme les boulangères, les blanchisseuses, les couturières, les gardiennes de bétail et les autres domestiques. Nous ignorons le nombre total de ce personnel, mais les registres fournissent des informations sur Marianne et Marie Texier, qui étaient rémunérées pour de telles tâches au fort Saint-Frédéric. Marianne était payée pour la boulange, la couture, le soin du bétail et le ménage, une combinaison de travaux d'intérieur et d'extérieur typique du travail des femmes à l'ère pré-industrielle. Au fort Frédéric, madame Courval gagnait 645 livres, pour des raisons inconnues. Marie-Joseph Baudria faisait le pain et la lessive au fort Frontenac, tandis qu'Angélique Le Pailleur faisait divers travaux de couture pour le commerce du Roi à Niagara ${ }^{70}$. Il est clair que

68. BAC, C11A 1747, vol. 117, fol. 443.

69. BAC, C11A, 1745, vol. 115, fol. 163, 187, 192 (Lafayette, Magnon); fol. 165-186 (L’Esperance). Les cornets de tissu entraient dans la composition des coiffes.

70. BAC, C11A, 1740, vol. 114, fol. 274v; 1741, fol. 274, 295v; vol. 75, fol. 325. Sur les efforts de la Couronne pour réduire le nombre de femmes aux postes, voir BAC, C11A, 1741, vol. 75, fol. 324-328, 1742, vol. 77, fol. 344-351v. Les Texier sont mentionnés dans BAC, C11A, 1740, vol. 114 , fol. 296v; 1747, vol. 115, fol. 218 ; 1746, vol. 85, fol. 329 et suivants; et: fol. 392-394; 1747, vol. 88, fol. 246; et dans l'article de Kathryn Young sur les négociantes. La responsable du ménage au fort Saint-Frédéric recevait la même paie que le barbier (60 livres l'an). Cela révèle-t-il le statut élevé des femmes, ou la piètre qualité des coupes de cheveux militaires? Indice de la polyvalence de ces femmes, Marianne Texier était aussi rémunérée en tant que couturière; voir BAC, C11A, vol. 77 , fol. $393 \mathrm{v}$. 
nombre de femmes se chargeaient des travaux de ferme, trayaient, faisaient le pain, s'occupaient de la lessive et cousaient pour les Français dans leurs forts.

Là encore les femmes à l'œuvre n’étaient pas nécessairement des «déléguées du mari». Au Domaine royal, le père missionnaire Claude Godefroi Coquart mentionne les trois filles, manifestement célibataires, de Joseph Dufour. Ces trois sœurs étaient payées pour élever des veaux et des agneaux, pour baratter et pour accomplir d'autres tâches ménagères qui les tenaient occupées du matin au soir ${ }^{71}$.

Même quand il y avait bel et bien un mari, il est difficile de déterminer jusqu'à quel point les activités mercantiles représentaient un travail d'équipe du couple. Et même quand il y avait équipe, ce n'est pas clair qui prenait les décisions. En écho à une observation faite des décennies plus tôt par le conseiller Martin de Lino à propos des femmes en affaires, l'intendant Hocquart écrivit en 1737 que beaucoup de femmes d'affaires gouvernaient les affaires de leur mari ${ }^{72}$. Moralisateur, Hocquart l'attribuait au fait que les hommes d'affaires et les officiers étaient attirés par le jeu et les autres divertissements; et il ajoutait qu'il trouvait que les femmes de "presque toutes les rang" faisaient preuve d'un esprit qui leur donnait une certaine supériorité sur leurs maris. Il ajoutait encore que les hommes à la campagne ne décidaient rien d'important sans consulter leurs femmes. Clairement, le mariage ne bannissait pas les épouses des hommes d'affaires ni celles des habitants de la table de négociation.

\section{NONNES EN GUERRE}

En plus des participantes individuelles, il y avait des communautés entières de femmes célibataires qui fournissaient des services essentiels en temps de guerre. De plus en plus d'études portent sur les activités économiques diversifiées des ordres religieux ${ }^{73}$. Ceux-ci avaient de l'argent à dépenser

71. Voir: «Memoir of Father Claude Godefroi Coquart on the Posts of the King's Domain» (1750), cité par K. Lawn et C. Salvucci, op. cit., 290.

72. Pour la remarque de Hocquart, voir la note insérée dans BAC, C11A, 1737, vol. 67, fol. $95 \mathrm{ff}$. Certaines épouses d'officiers servant dans les forts aux frontières recevaient la moitié de la solde de leur mari, sans doute pour les aider à gérer la seigneurie ou à faire face à d'autres dépenses. Voir J. Brun, op. cit., 24, pour l'affirmation de Martin de Lino au début du XviII ${ }^{e}$ siècle, selon laquelle «la majeure partie des membres du conseil font faire leur commerce par leurs femmes ou leurs enfants». Cela suggère que les épouses étaient actives mais non de leur propre initiative, alors que Hocquart relève explicitement leur gouvernance active.

73. Voir, par exemple, les travaux de Micheline D’Allaire sur les ordres religieux féminins, tout récemment ses deux volumes sur Les communautés religieuses de Montréal (Montréal, Méridien, 1997 et 2002); les deux volumes de Francois Rousseau, La Croix et le scalpel: Histoire des Augustines 
en travail et en fournitures, et tout un assortiment de denrées à vendre. Leur participation était facilitée par la parenté et les liens de clientélisme avec le gouvernement et les groupes de marchands. La guerre provoquait normalement une augmentation des paiements aux sœurs pour les soins qu'elles prodiguaient aux soldats hospitalisés, une source de revenus substantielle pour les deux hospices et les trois hôpitaux à Québec, TroisRivières et Montréal. Étonnamment, les chantiers navals aussi rapportaient de l'argent aux moniales à l'Hôtel-Dieu de Québec. Durant les années 1740, le couvent figurait chaque année dans les comptes de la Couronne comme fournissant à la fois de la nourriture et des médicaments aux ouvriers. L'Hôtel-Dieu et l'Hôpital Général étaient également dédommagés pour les repas servis dans leurs salles aux charpentiers et marins malades ou blessés. Mais les livraisons de l'Hôtel-Dieu, constituées d'oignon, d'échalote, d'ail, de laitue, de persil et d'autres herbes pour les équipages et les ouvriers des chantiers navals, semblent avoir été des lots. Allaient-ils aux travailleurs dans leurs quartiers, ou étaient-ils entreposés pour les navires? Y avait-il une destination similaire pour les treize sirops différents et autres remèdes attribués à madame de Saint-Jean, apothicaire de l'Hôtel-Dieu, le tout en un seul lot, le 31 juillet $1745^{74}$ ? Les sœurs ayant une formation en pharmacie faisaient des remèdes pour les vendre et les expédiaient aussi loin qu'aux forts Chambly, Frontenac, Niagara et Détroit $^{75}$. Comme nous l'avons vu, les sœurs grises de Montréal faisaient leur propre contribution en uniformes et tentes pour les hommes qui patrouillaient aux frontières. D'innombrables mains étaient à l'ouvrage dans les couvents et fabriquaient de quoi vendre.

\section{CONCLUSION}

Cloîtrées ou laïques, mariées ou célibataires, les femmes canadiennes tirèrent profit des ressources du pays. N'ayant souvent aucun mari à

et de l'Hôtel Dieu de Québec, 1639-1989 (Sillery, Septentrion, 1989 et 1994); Jan Noel, "Caste and Clientage in an Eighteenth Century Québec Convent", Canadian Historical Review, 82 (2001): 27-41; et Colleen Gray, The Congrégation de Notre-Dame, Superiors, and the Paradox of Power, 16931796 (Montréal, McGill-Queen's University Press, 2007), 250 p.

74. BAC, C11A, 1743, vol. 80, fol. 186; 1745, vol. 83, fol. 248, vol. 84, fol. $77 ; 1748$, vol. 92 , fol. 201. En d'autres cas, il fut spécifié, une fois pour l'Hôpital Général en 1745, que les repas seraient fournis aux équipages, charpentiers et autres ouvriers qui étaient des patients. La rémunération de mère Sainte-Geneviève de l'Enfant Jésus pour des repas servis aux marins malades entre 1733 et 1736 est enregistrée dans BAC, C11A, 1736, vol. 68, fol. 46.

75. BAC, C11A, 1731, vol. 56, fol. 178-179v; 1732, vol. 58, fol. 38-41; 1740, vol. 114, fol. 288; 1745, vol. 115, fol. 207. 
consulter, elles ne se bornèrent pas à être les auxiliaires maritales des désirs masculins, réels ou imaginaires. Comme le suggèrent les commentaires de l'intendant Hocquart et comme le confirment les comptes de la traite des fourrures et de la Couronne, nous devons cesser de faire de l'activité commerciale du mari la norme et de celle de l'épouse l'exception, afin de reconnaitre à quel point il était usuel dans une économie fondée sur l'exploitation des matières premières de faire un peu de négoce. Les nombreuses contributions et activités de femmes de toutes les classes et de tous les statuts suggèrent qu'une conception plus large est nécessaire.

Qu'est-ce qui ne va pas avec les termes en usage actuellement? La traite des fourrures et l'économie de guerre suscitèrent une variété de contributions économiques par une telle variété de femmes qu'elles rendent inadéquat le concept de délégation. Les données indiquent qu'elles furent nombreuses à fournir de leur propre chef des marchandises, des produits manufacturés ou des services. Plus fondamentalement, même si les sources ne révèlent pas le statut de toutes ces femmes, beaucoup sont explicitement consignées comme des veuves ou demoiselles, ce qui signifie qu'elles n'avaient tout simplement pas de mari pour les «déléguer». Quant à la proportion, qu'un notaire ait enregistré leurs activités ou non, c'était une pratique quotidienne et ordinaire pour les femmes canadiennes de produire des biens et de les mettre en marché.

Les sources révèlent plus qu'une poignée de femmes exceptionnelles. Elles étaient trop bien réparties dans toute la colonie (dans toutes les classes et les ethnies, de plus) pour constituer un quelconque "réseau» féminin dont les membres se seraient soutenues mutuellement. Elles nous mettent sur la piste d'une réalité que les historiens européens ont depuis longtemps remarquée, mais que nous avons tendance à oublier parce que tant de ces activités quotidiennes, accomplies par des personnes pauvres et illettrées qui n'auraient jamais songé à entrer chez un notaire, n'ont été enregistrées nulle part. En France et en Angleterre, au début de l'ère moderne, ainsi que l'ont démontré Olwen Hufton, Dominique Godineau, Daryl Hafter, Peter Earle et bien d'autres auteurs, la production et le commerce de biens ou de services étaient monnaie courante chez les femmes de toutes classes. Hommes, femmes et enfants travaillaient parfois de concert, parfois séparément pour accomplir des tâches plus spécifiques à un âge ou à un sexe. Tout le monde était censé contribuer, même les enfants. À cause de l'absence des hommes, éloignés par la traite ou la guerre, cette dépendance de l'économie familiale pré-industrielle a pu être accentuée au Canada (comme elle l'a été dans certaines provinces de 
France, telle l'Auvergne, que les hommes quittaient en quête de travail). Nos exemples attestent que les femmes de l'élite comme du peuple trouvaient toutes sortes de façons de s'insérer dans le cycle économique fait de production et d'échange. L'intendant Hocquart accorde même quelque appui à l'affirmation, difficilement prouvable, que c'était plus courant en Nouvelle-France qu'en France même, quand il souligne la prédilection des Canadiennes pour le commerce.

Les publications sur les femmes en Nouvelle-France, qui ont privilégié les chefs d'entreprise, ont négligé la nature quotidienne des échanges économiques féminins. Il serait crucial de transcender la dichotomie entre "femmes d'affaires» et "ménagères». Quelques négociantes très actives sont parfois désignées par les historiens comme des "femmes d'affaires» ou des chefs d'entreprise; et il y en a eu, comme les Pascaud, Fornel, Ramezay et Desauniers. Mais la traite des fourrures et les commandes militaires dont nous avons parlé ici annexent au monde de l'écrit un groupe nettement plus vaste qui participait à l'économie monétaire d'une manière moins systématique. Elles ménagent des aperçus d'un travail féminin plus prosaïque et plus fragmenté. Les historiens européens ont mis en lumière cette dimension de la réalité féminine au cours de ces dernières décennies, mais les universitaires canadiens en ont rarement parlé. Il nous faut intégrer cette participation féminine omniprésente à la vie commerciale de la Nouvelle-France dans nos discussions du xxI ${ }^{\mathrm{e}}$ siècle, afin de la reconnaître comme la norme et non l'exception.

À y regarder de près, il semble que les héroïnes de l’abbé Groulx «qui ont fait aller berceau après berceau, et, qui, près de ces berceaux, ont toujours trouvé le courage de rire et de chanter ${ }^{76}$ » étaient vraisemblablement en train de compter aussi bien les pièces de monnaie que les bébés ou les neuvaines. Comme l'a fait remarquer la médiéviste Judith Bennett, les femmes «tended to be intermittent workers, jumping from job to job or juggling several tasks at once. This was true in 1300, and it remained true in $1700 .{ }^{77}$ ». Alice Clark le formule de façon plus positive en notant que «owing to the greater complexity of a woman's life her productive capacity must be classified on different lines from those which are generally followed in dealing with the economic life of $\operatorname{men}^{78}$ ». Si nous ne tenons pas compte de cette complexité et faisons des femmes ordinaires

76. Lionel Groulx, La Canadienne Française (Nicolet, n. p., 1950), 9.

77. Voir Judith Bennett, «Medieval Women, Modern Women: Across the Great Divide», dans David Aers, dir., Culture and History, 1350-1600 (Detroit, Wayne State University Press, 1992), 158.

78. Voir A. Clark, op. cit., 4. 
des femmes au foyer, à la ferme et à l'église, nous omettons leur présence des plus réelles dans l'économie de marché.

Plus fréquemment qu'on ne tombe sur des Pascaud et des Desauniers, on retrouve des commerçantes modestes, parfois intermittentes et souvent mariées avec enfants. De tout temps les mères ont été polyvalentes. La séparation rigide des occupations socioprofessionnelles s'applique à des époques plus récentes, et plus souvent aux hommes ${ }^{79}$. Si les épouses de la colonie ne montaient une échoppe ou ne recevaient des gages annuels qu'à l'occasion, un grand nombre d'entre elles produisaient quelque chose qui se retrouvait sur le marché. Des Canadiennes cousant à la maison des articles pour la traite des fourrures sont mentionnées dès le $\mathrm{xvII}^{\mathrm{e}}$ siècle; des marchandes canadiennes apparaissent dans les illustrations et les descriptions des marchés urbains pendant de nombreuses décennies après la période en question. Les femmes adultes n'étaient pas "dépendantes", et elles ne faisaient pas quelque chose d' "exceptionnel» quand, tout en élevant une famille, elles négociaient avec un Indien de passage, livrait des oignons et de l'eau potable aux hommes des chantiers navals, passaient contrat pour faire la blanchisserie du régiment, vendaient le bois dont elles pouvaient disposer ou passaient les soirées d'hiver à faire des chemises que des Autochtones amis enfileraient des milliers de kilomètres plus à l'ouest.

La colonie était pauvre, comme l'ont souvent noté les officiels français. Les colons faisaient tout leur possible pour gagner un peu d'argent, ouverts aux occasions de trafiquer et impatients de servir quand la guerre ouvrait grand les coffres du Trésor français. Au xviII ${ }^{\mathrm{e}}$ siècle, les femmes de la vallée laurentienne travaillaient sur les berges et sur les fermes, s'occupaient de leurs étals au marché, tenaient des échoppes d'artisans et s'affairaient dans les jardins, boulangeries et pharmacies des couvents. Elles surgissent fugitivement dans les registres des intendants, des voyageurs et des notaires. Durant les années 1740, elles sortent de l'ombre en grand nombre, leurs noms s'alignant les uns après les autres dans les comptes de guerre de la Couronne. Il en ressort clairement que la commerçante était plus modeste que la grande dame que l'on retrouve dans les études précédentes sur le sujet. Si elle n'était pas particulièrement favorisée, elle avait néanmoins quelque chose de commercialisable, et elle

79. Il est vrai que les hommes aussi pouvaient proposer des services variés, par exemple Étienne Blot qui émargeait à la Couronne en tant que charpentier, charretier et fournisseur de menus articles dont le bois et les chandelles (ce dernier article étant peut-être une contribution cachée de son épouse). Voir Catherine Desbarats, loc. cit., 199. 
en attendait une compensation. On ne peut reléguer la mulier œeconomica en Nouvelle-France en marge de la société. Partenaire fiable de l'homo aconomicus, elle aurait été pratiquement toutes les femmes dans une économie pré-industrielle à forte intensité de main-d'œuvre où même les épouses d'officiers devaient grappiller un peu d'argent. Les exceptions n'étaient pas celles qui négociaient, mais celles qui ne le faisaient pas.

Traduction: Jean-Louis Trudel 